\title{
Synthesis of spiro-cyclohexadienone- $\gamma$-lactams via free-radicals and study of thermal regioselective spirocyclization
}

\author{
Pedro A. Cano ${ }^{1,2}$, Alejandro Islas-Jácome ${ }^{3}$ and Rocío Gámez-Montaño ${ }^{1, *}$ \\ 1 Departamento de Química, División de Ciencias Naturales y Exactas, Universidad de Guanajuato, Noria \\ Alta S/N, Col. Noria Alta, Guanajuato, C.P. 36050, Gto. México. \\ 2 Departamento de Ingeniería Civil, División de Ingenierías, Universidad de Guanajuato, Av. Juárez No. 77, \\ Zona Centro, Guanajuato, C.P. 36000, Gto., México. \\ 3 Departamento de Química, Universidad Autónoma Metropolitana-Iztapalapa, San Rafael Atlixco 186, Col. \\ Vicentina, Del. Iztapalapa, C.P. 09340, Ciudad de México. \\ * Correspondence: rociogm@ugto.mx; Tel.: +52-473-73-20006, ext. 8191
}

Academic Editor: Julio A. Seijas

Received: date; Accepted: date; Published: date

\begin{abstract}
The spiro-regioisomers $\mathbf{1 a}$ and $\mathbf{1 b}$ were synthesized from the xanthate-type precursor $\mathbf{2}$ via a free-radical mediated spirocyclization in different proportions depending on the reaction temperature, from $1: 40$ at $-45^{\circ} \mathrm{C}$ to $1: 5$ at $150{ }^{\circ} \mathrm{C}$. The maximum proportion relationship value was 1:2 at $110-125{ }^{\circ} \mathrm{C}$. The regioselectivity was a result from the inherent stability of the involved intermediate free-radicals. The intermediate of the regioisomer $\mathbf{1 b}$ is a very thermodynamically stable double allyl radical and for regioisomer $\mathbf{1 a}$, its intermediate is an $\alpha, \beta, \gamma, \delta$-unsaturated carbonyl radical. The products $\mathbf{1 a}-\mathbf{b}$ were fully characterized by ${ }^{1} \mathrm{H}$ and ${ }^{13} \mathrm{C}$ NMR, even by $\mathrm{X}$-ray analysis because adequate crystals were obtained for both products, (1a, CCDC: 1050852) and (1b, CCDC: 1050853), respectively.
\end{abstract}

Keywords: Free-radicals, Xanthates, Spirocyclization, Regioselectivity,

\section{Introduction}

The spirocyclic moiety is the main structural core of various natural and synthetic bioactive molecules, pro-drugs and drugs [1-2]. Particularly, the azaspirocyclohexendienones are the core also and very important intermediates toward the synthesis of biologically active molecules [3]. The figure $\mathbf{1}$ shows the structure of the alkaloid Annosqualine $\mathbf{0}$ (isolated from Annona squamosa) [4]. It is noteworthy that the regioisomer $\mathbf{1 b}$ has the same azaspirocyclic core than the natural product $\mathbf{0}$, which additionally has a tetrahydroisoquinoline framework in fused manner. With respect to the azaspirocyclic nucleus of the compound 1a, there is no reports (as far as we know) about its synthesis and/or related products exhibiting pharmacological activity.
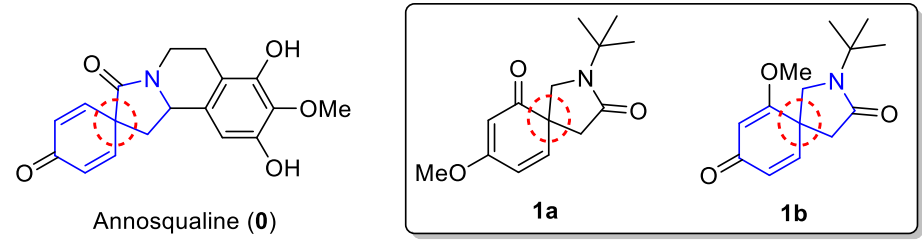

Figure 1. Natural and synthetic spiroheterocycles 
The synthesis of spiro-cyclohexadienone- $\gamma$-lactams is a poorly explored research area. For example, the synthesis via free-radical spirocyclization onto a $p$-MeO-aryl rings was first reported in 1967 by D.H. Hey [5]. However, such process has a limited synthetic value. In the same way, only two further works by S.Z. Zard [6] and D.G. Curran [7] report similar methodologies. Finally, in 2007 we reported a novel related methodology [8].

Besides, there are two general approaches toward the synthesis of spiro-cyclohexadienone type compounds: i) de-aromatization of the involved $p$-MeO-benzene moieties, and ii) intramolecular spirocyclization. Indeed, in this year, C.R. Reddy and co-workers reviewed masterfully the most recent advances on the synthesis of spyro-cyclohexendienone- $\gamma$-lactams and other related spiroheterocycles via ionic and free-radical mediated ipso-spirocyclizations [9]. Thus, we herein report a study of regioselectivity via free-radical mediated spirocyclization toward the synthesis of a couple of spiro-cyclohexendienone- $\gamma$-lactams from a xanthate precursor, which was synthesize stepwise.

\section{Results and discussion}

Our initial hypothesis behind the present work was that the spiroheterocyclic compound $\mathbf{1 b}$ could be synthesized easily via free-radical mediated 6-endo-trig ipso-spirocyclization from the corresponding 2,4-dimethoxyphenyl-containing xanthate 2, table 1 . Thus, we took as starting point the standard conditions for this kind of free-radical mediated reactions (as reported by us [8]). DLP was used as both, free radical initiator and oxidant [10], while the 1,2-dichloroethane was used as solvent. However, together with $\mathbf{1 b}$, we found its spiro-regioisomer $\mathbf{1 a}$ as by-product. Then, motivated by this finding, we performed a study to synthesize in selective manner each regioisomer. In this context, we chose the parameter temperature $(T)$ as pivotal point to perform our study.

Of course, DLP needs high temperature to make its O-O bond cleavage. In order to carry out our first attempts under kinetic conditions, we needed a free radical initiator capable with low temperatures, so triethyl boron was used to perform the reactions at $-45,-25,0$ and $25^{\circ} \mathrm{C}$, respectively. As seen, both spiro-regioisomers were obtained in 1:40, 1:20, 1:13 and 1:7 proportion relationships, respectively. Then, because the proportion became closer by increasing the temperature, we used again DLP to perform further experiments at $90,110,125$ and $150{ }^{\circ} \mathrm{C}$ observing relationship values of 1:4, 1:2, 1:2 and 1:5, respectively, table 1 .

Table 1. Study of thermal spirocyclization

\begin{tabular}{|c|c|c|c|}
\hline 2 & & & \\
\hline$T\left({ }^{\circ} \mathrm{C}\right)$ & $1 a^{a}$ & $1 b^{a}$ & $\% \mathbf{o}^{\mathrm{b}}$ \\
\hline-45 & 1 & 40 & 77 \\
\hline-25 & 1 & 20 & 78 \\
\hline 0 & 1 & 13 & 82 \\
\hline 25 & 1 & 7 & 80 \\
\hline 90 & 1 & 4 & 88 \\
\hline 110 or 125 & 1 & 2 & 92 \\
\hline 150 & 1 & 5 & 82 \\
\hline
\end{tabular}

a Relationship values determined by H NMR

${ }^{b}$ Yields $(\mathbf{1 a}+\mathbf{1 b})$ measured after purification by silica-gel column chromatography 
As seen, the maximum proportion relationship value was $1: 2$ at 110 or $125^{\circ} \mathrm{C}$. We hypothesize that proportion values are due to inherent stability of the involved free radicals. In the case of the product $\mathbf{1 b}$, its intermediate $\mathbf{4 b}$ is a very thermodynamically stable double allyl radical. In the case of the product 1a, the intermediate $4 \mathbf{a}$ is an $\alpha, \beta, \gamma, \delta$-unsaturated carbonyl radical. The scheme 2 shows the more plausible reaction mechanisms.

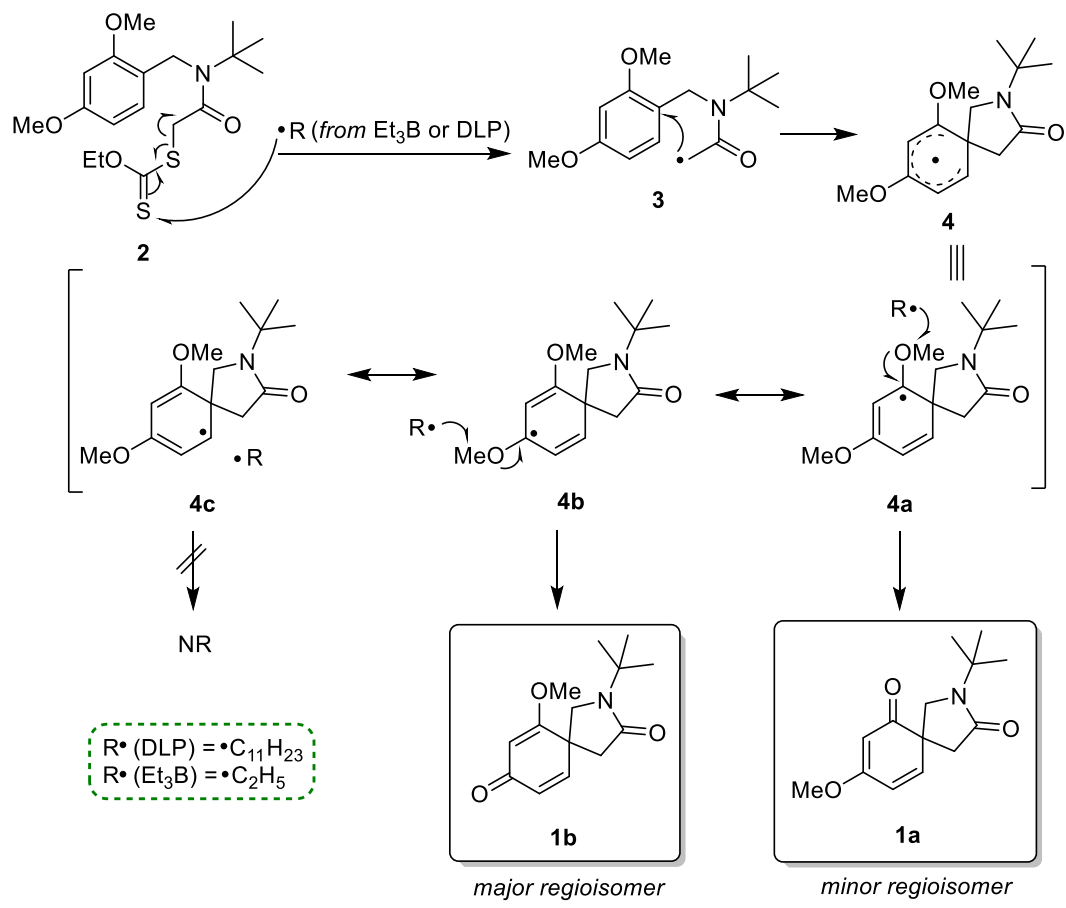

Scheme 2. Mechanism of the free-radical mediated spyrocyclization

As it was expected, the conjugated free-radical $4 \mathrm{c}$ did not give any product because the probability to find the electron in such position is practically inexistent. In fact, the electron of the free-radicals $4 \mathbf{a}$ and $\mathbf{4 b}$ are strongly stabilized by resonance. In addition, the attack of $\mathrm{R} \cdot$ to methyl (of methoxy group) in the intermediate $4 \mathbf{b}$ occurs easier than in $\mathbf{4 a}$ because there is lower associated steric hindrance coming from the merged pyrrolodinone ring. The spiroregioisomers $\mathbf{1 a}$ and $\mathbf{1 b}$ were suitably characterized. Thus, we show the H and C NMR spectra, even their X-ray ORTEPs, figure 2.

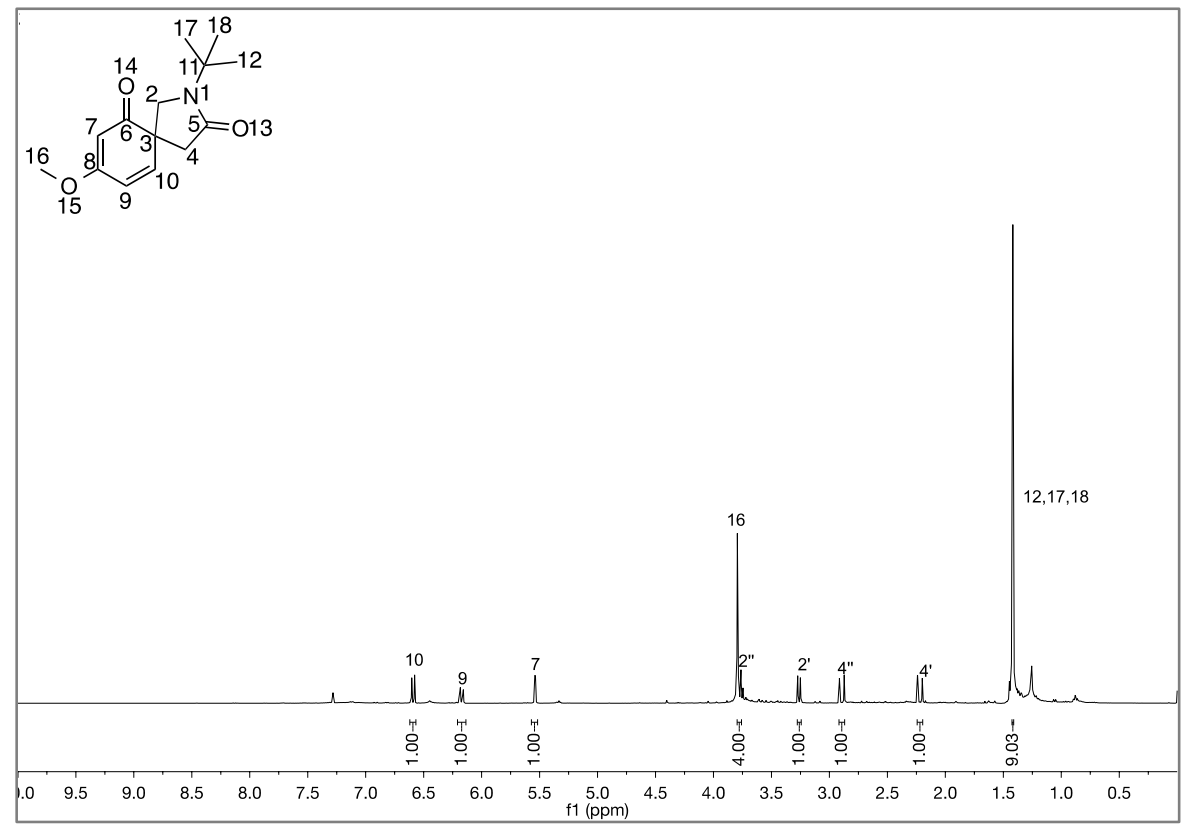

Figure 2a. ${ }^{1} \mathrm{H}$ NMR spectrum of the spiroregioisomer 1a 


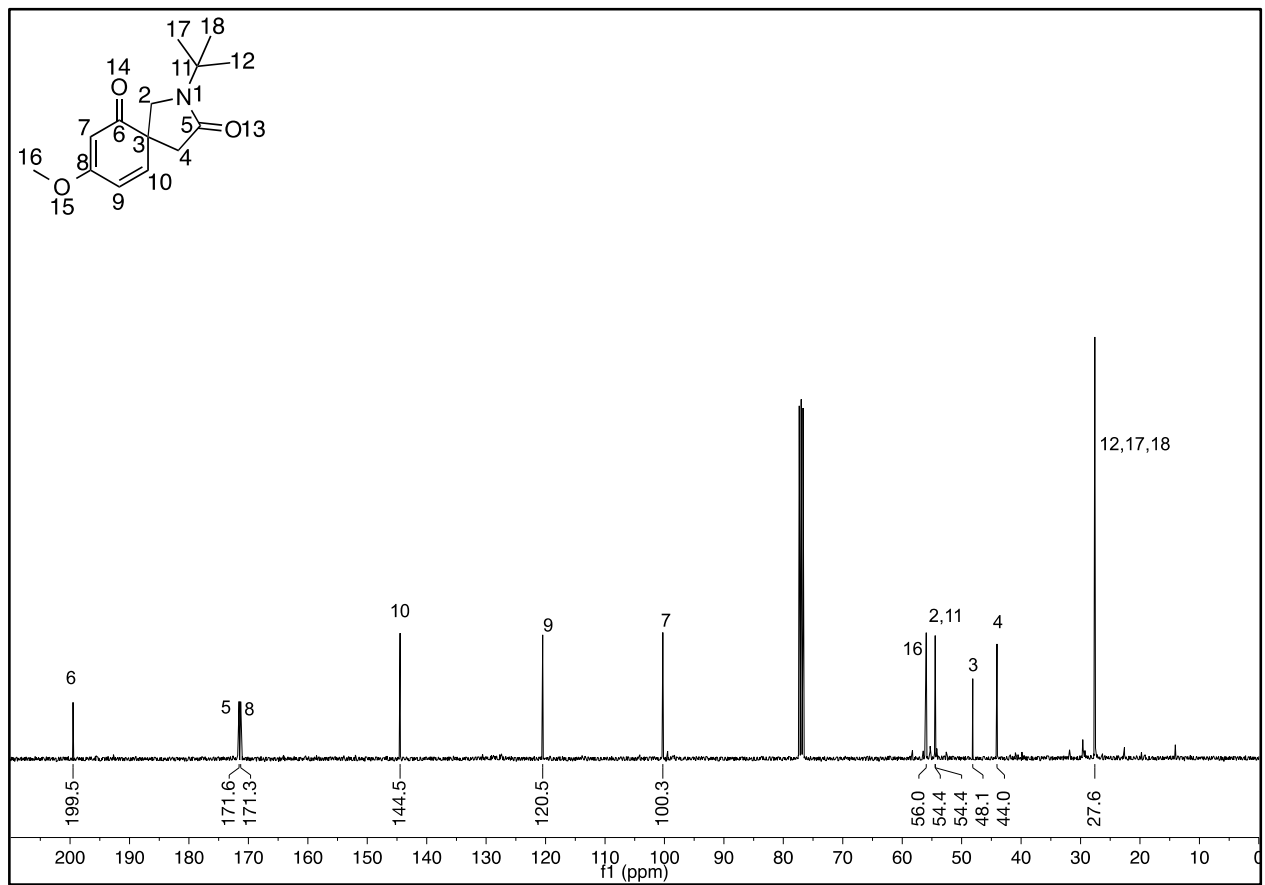

Figure $\mathbf{2 b} .{ }^{13} \mathrm{C}$ NMR spectrum of the spiroregioisomer $\mathbf{1 a}$

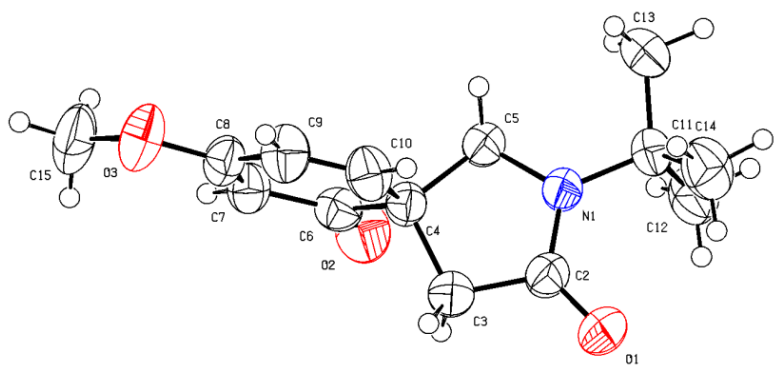

Figure 2c. ORTEP of the spiroregioisomer 1a (CCDC: 1050852)

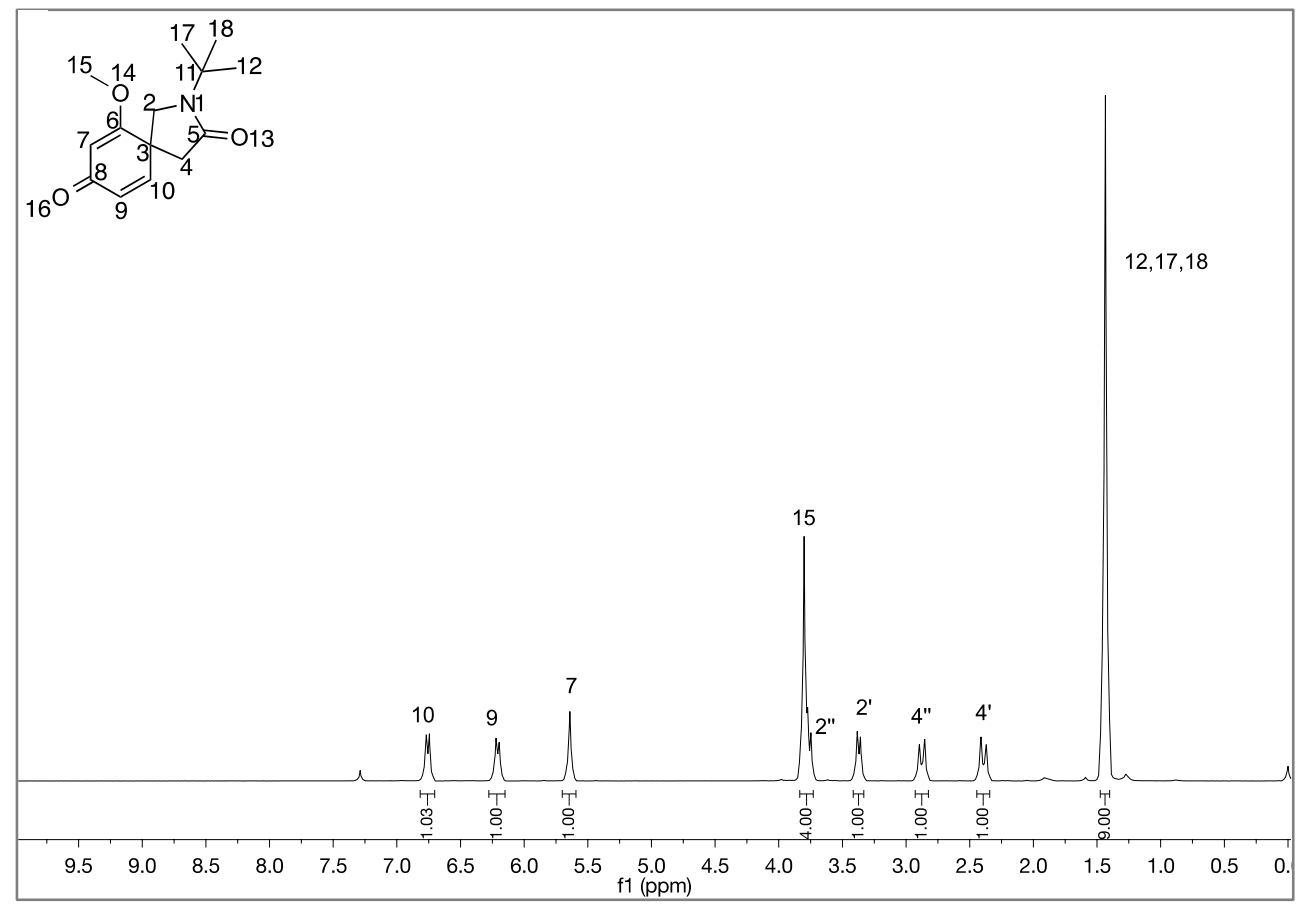

Figure $2 \mathrm{~d}^{1} \mathrm{H}$ NMR spectrum of the spiroregioisomer $\mathbf{1 b}$ 


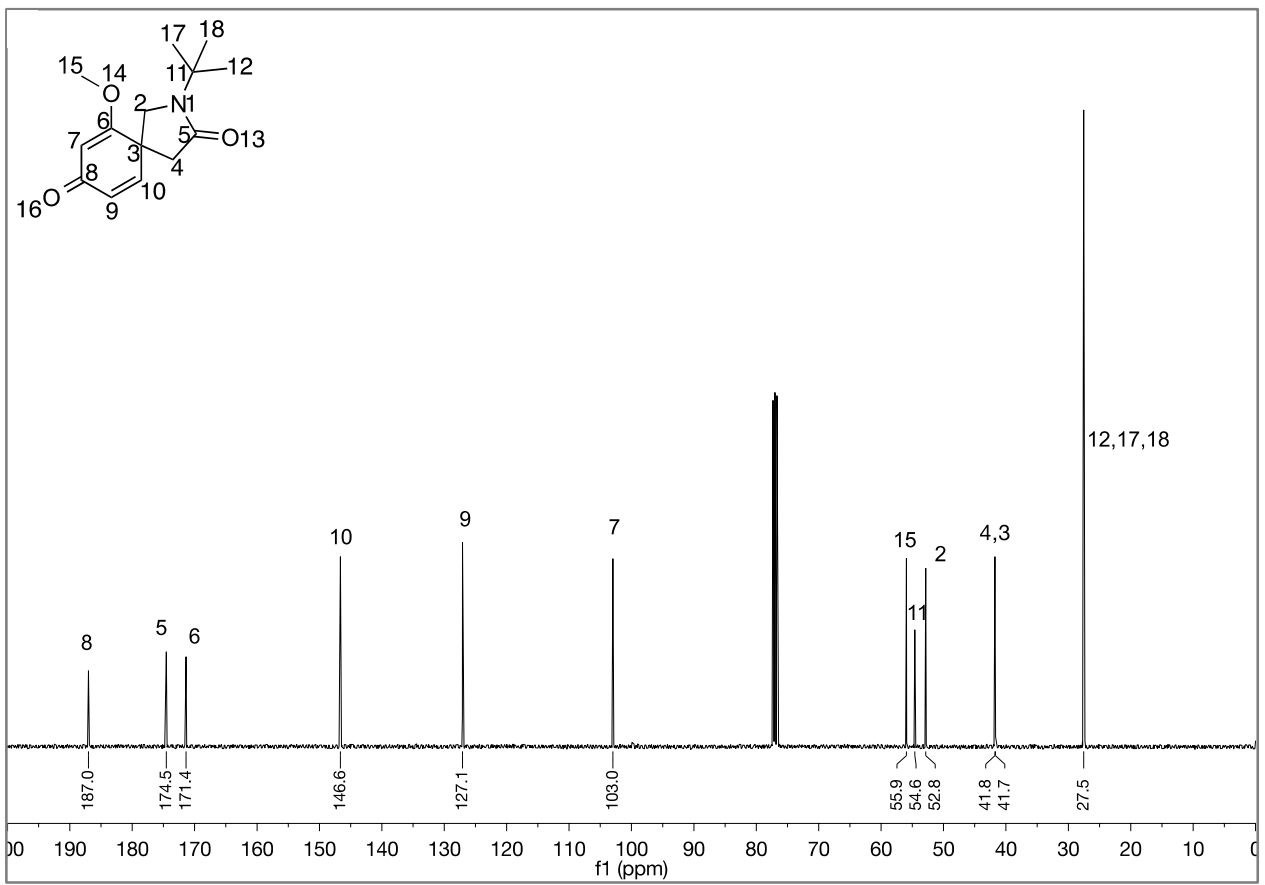

Figure 2 e. ${ }^{13} \mathrm{C}$ NMR spectrum of the spiroregioisomer $\mathbf{1 b}$

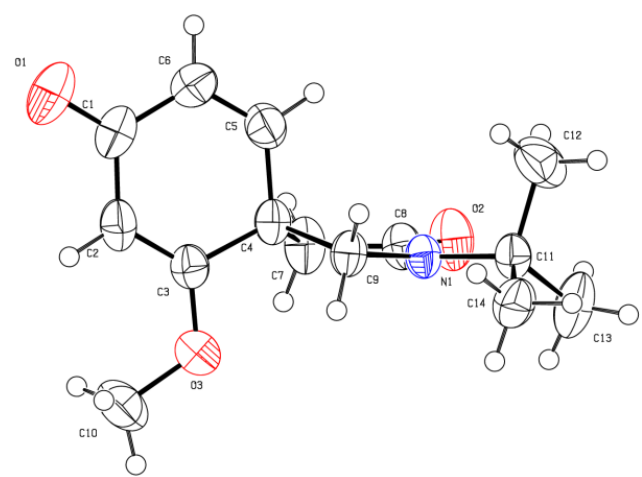

Figure 2f. ORTEP of the spiroregioisomer 1b (CCDC: 1050853)

\section{Conclusion}

The azaspiro-heterocycle $\mathbf{1 b}$ was synthesized in minor proportion with respect to its regioisomer 1a probably due to stereoelectronic factors: $i$ ) stability of its involved intermediate (a double allyl versus an $\alpha, \beta, \gamma, \delta$-unsaturated), and ii) the steric hindrance coming from the adjacent pyrrolidinone ring. The ORTEPs confirmed unequivocally their structures. This communication represents a contribution toward azaspiro-ciclohexendienones, and the first work describing the synthesis of a cyclohexendienone containing an ortho-carbonyl moiety to a spirocenter.

\section{Experimental part}

\section{General information, instrumentation and chemicals}

${ }^{1} \mathrm{H}$ and ${ }^{13} \mathrm{C}$ NMR spectra were acquired on either, Bruker Advance III (500 or $400 \mathrm{MHz}$ ) spectrometers. The solvent was deuterated chloroform $\left(\mathrm{CDCl}_{3}\right)$. Chemical shifts are reported in parts per million $(\delta / \mathrm{ppm})$. Internal reference for ${ }^{1} \mathrm{H}$ NMR spectra is respect to TMS at $0.0 \mathrm{ppm}$. Internal reference for ${ }^{13} \mathrm{C}$ NMR spectra is respect to $\mathrm{CDCl}_{3}$ at $77.0 \mathrm{ppm}$. Coupling constants are reported in Hertz (J/Hz). Multiplicities of the signals are reported using the standard abbreviations: singlet (s), 
doublet (d), triplet (t), quartet (q) and multiplet (m). IR spectra were acquired on a Bruker Tensor 27 spectrophotometer. The absorbance peaks are reported in reciprocal centimeters $\left(\mathrm{cm}^{-1}\right)$. Reaction progress was monitored by TLC on precoated Kieselgel $60 \mathrm{~F}_{254}$ plates and the spots were visualized under UV light (254 or $365 \mathrm{~nm}$ ). Flash column chromatography was performed using silica gel (230400 mesh) and a mixture of hexanes with AcOEt $(4: 1$ or $7: 3 \mathrm{~V} / \mathrm{V})$ as mobile phase. A mixture of hexanes with either, $\mathrm{DCM}$ with $\mathrm{CHCl}_{3}(9: 1 \mathrm{~V} / \mathrm{V})$ was used as recrystallization solvent. Melting points were determined on a Fisher-Johns apparatus and were uncorrected. All starting materials were purchased from Sigma-Aldrich and were used without further purification. The solvents were distilled and dried according to standard procedures.

\section{Synthesis and characterization of the xanthate 2}

In a round-bottomed flask equipped with a magnetic stirring bar, to a $0.5 \mathrm{M}$ solution of 2,4dimethoxyaldehyde (1.0 equiv.) in anhydrous $\mathrm{MeOH}$ under nitrogen atmosphere at room temperature, the tert-butylamine (1.1 equiv.) and molecular sieves ( $4 \AA$ ) were added. After 30 minutes, imine solution was placed into an ice bath $0^{\circ} \mathrm{C}$ and added $\mathrm{NaBH}_{4}$ (1.0 equiv.). Resulting amine mixture was filtered over a celite pad and the solvent was evaporated under reduced pressure. Residue was dissolved in AcOEt and washed with distilled water, dried over $\mathrm{Na}_{2} \mathrm{SO}_{4}$ and concentrated in vacuum to obtain the amine in $98 \%$ yield, which was used in the next step without further purification.

To the amine solution in dichloromethane, was added trimethylamine (2.0 equiv.) and placed at $0^{\circ} \mathrm{C}$ on iced bath. Slowly, was added 2-chloroacetic acid (1.1 equiv). After 15 minutes reacting at room temperature, the solvent was evaporated, and the residue was dissolved in AcOEt and washed with distilled water, dried with $\mathrm{Na}_{2} \mathrm{SO}_{4}$ and concentrated in vacuum to obtain the corresponding chloride in $80 \%$ yield, which was used in the next step without further purification.

To the chloride solution in $\mathrm{MeOH}$, was added potassium salt of xanthic acid (1.1 equiv). After 1 $\mathrm{h}$, the reaction mixture was filtered. The solvent was removed under reduced pressure until dryness. The residue was dissolved in EtOAc and washed with distilled water. The combined organic layer was dried over anhydrous $\mathrm{Na}_{2} \mathrm{SO}_{4}$. The resulting yellowish crude was immediately purified by silica gel column chromatography to obtain the xanthate 2 in $90 \%$ yield.

$N$-tert-butyl- $N$-[(2,4-dimethoxyphenyl)methyl]-2-[(ethoxymethanethioyl)sulfanyl] acetamide (2): Physical appeareance: yellow oil, $R_{f}: 0.42$ (Hex-EtOAc, 4/1, v/v), IR: 1610 (C=S), ${ }^{1} \mathrm{H}$ NMR (500 $\left.\mathrm{MHz}, \mathrm{CDCl}_{3}\right): \delta 7.16(\mathrm{~d}, J=8.2 \mathrm{~Hz}, 1 \mathrm{H}, \mathrm{H}-3), 6.50$ (d, J= $\left.8.3 \mathrm{~Hz}, 1 \mathrm{H}, \mathrm{H}-4\right), 6.47$ (s, 1H, H-6), 4.60 (d, $J=$ $12.1 \mathrm{~Hz}, 4 \mathrm{H}, \mathrm{H}-9, \mathrm{H}-29), 4.03$ (s, 2H, H-19), 3.83 (s, 3H, H-14), 3.81 (s, 3H, H-8), 1.44 (s, 9H, H-12, H17, H-18), 1.37 (t, J = 6.7 Hz, 3H, H-25), ${ }^{13} \mathrm{C}$ NMR (126 MHz, CDCl 3$)$ ठ 214.0 (C-21), 167.6 (C-15), 160.0 (C-1), 156.9 (C-5), 126.8 (C-3), 118.8 (C-2), 103.8 (C-4), 98.4 (C-6), 70.0 (C-24), 58.4 (C-11), 55.3 (C-14), 55.2 (C-8), 44.1(C-9), 41.9 (C-19), 28.3 (C-12, C-17, C-18), 13.6 (C-25).

Typical procedure for DLP-assisted free-radical spirocyclization. A solution of the corresponding xanthate $2(1.0 \mathrm{mmol})$ in toluene $[0.5 \mathrm{M}]$ was heated at reflux, and $10 \% \mathrm{~mol}$ lauryl peroxide $(1.5 \mathrm{mmol})$ was then portion wise added every $1 \mathrm{~h}$ until complete consumption of the xanthate was observed by TLC. The solvent was removed under reduced pressure and the residue was purified by chromatography on a silica gel column (EtOAc/Hex) to furnish the desired spirocyclic product.

Typical procedure for Et3B-assisted free-radical spirocyclization (low temperatures). A solution of $\mathrm{Et}_{3} \mathrm{~B}(1.0 \mathrm{M}$ in THF) was dropwise added (4 equiv./16 h) to a stirred solution of the xanthate $2(1.0$ $\mathrm{mmol}$ ), and $\mathrm{FeSO}_{4}(3.0 \mathrm{mmol})$ in $\mathrm{CH}_{2} \mathrm{Cl}_{2} / \mathrm{MeOH} / \mathrm{H}_{2} \mathrm{O}(1: 4: 2,4.0 \mathrm{~mL} / \mathrm{mmol}$ of the xanthate 2$)$. The solvent was removed under reduced pressure and the crude residue extracted with EtOAc. The organic layer was dried with $\mathrm{Na}_{2} \mathrm{SO}_{4}$, and solvent removed under reduced pressure. The product was purified by chromatography on a silica gel column (EtOAc/Hex) to afford the desired spyrocyclic product.

2-(tert-butyl)-8-methoxy-2-azaspiro[4.5]deca-7,9-diene-3,6-dione (1a): Physical appeareance: colorless crystals, mp: $219^{\circ} \mathrm{C}, \mathrm{R}_{f}: 0.36$ (Hex-EtOAc, 2/3, v/v), IR: $1669(\mathrm{C}=\mathrm{O})$ and $1651(\mathrm{C}=\mathrm{O}),{ }^{1} \mathrm{H}$ NMR $\left(400 \mathrm{MHz}, \mathrm{CDCl}_{3}\right) \delta 6.59$ (d, J=10.0 Hz, 1H, H-10), 6.17 (d, J=12.1 Hz, 1H, H-9), 5.54 (s, 1H, H-7), 3.80 
$-3.76\left(\mathrm{~m}, 4 \mathrm{H}, \mathrm{H}-16, \mathrm{H}-2^{\prime \prime}\right), 3.26$ (d, J = 9.6 Hz, 1H, H-2'), 2.89 (d, J=16.3 Hz, 1H, H-4"'), 2.22 (d, J = 16.3

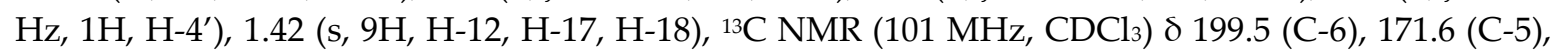
171.3 (C-8), 144.5 (C-10), 120.5 (C-9), 100.3 (C-7), 56.0 (C-16), 54.4 (C-2), 54.4 (C-11), 48.1 (C-3), 44.0 (C4), 27.6 (C-12, C-17, C-18), (CCDC: 1050852, these data can be obtained free of charge via http://www.ccdc.cam.ac.uk/conts/retrieving.html (or from the CCDC, 12 Union Road, Cambridge CB2 1EZ, UK; Fax: +441223 336033; E-mail: deposit@ccdc.cam.ac.uk)).

2-(tert-butyl)-6-methoxy-2-azaspiro[4.5]deca-6,9-diene-3,8-dione (1b): Physical appeareance: colorless crystals, mp: $228^{\circ} \mathrm{C}, \mathrm{R}_{f}$ : 0.30 (Hex-EtOAc, 2/3, v/v), IR: $1690(\mathrm{C}=\mathrm{O})$ and $1664(\mathrm{C}=\mathrm{O}),{ }^{1} \mathrm{H}$ NMR $\left(400 \mathrm{MHz}, \mathrm{CDCl}_{3}\right) \delta 6.76(\mathrm{~d}, J=9.9 \mathrm{~Hz}, 1 \mathrm{H}, \mathrm{H}-10), 6.21$ (d, J = 9.9 Hz, 1H, H-9), 5.64 (s, 1H, H-7), 3.79 (m, 4H, H-15, H-2'), 3.37 (d, J=10.0 Hz, 1H, H-2'), 2.88 (d, J = $\left.16.3 \mathrm{~Hz}, 1 \mathrm{H}, \mathrm{H}-4^{\prime \prime}\right), 2.39$ (d, J = $16.4 \mathrm{~Hz}$, 1H, H-4'), 1.43 (s, 9H, H-12, H-17, H-18), ${ }^{13} \mathrm{C} \mathrm{NMR} \mathrm{(101} \mathrm{MHz,} \mathrm{CDCl} 3$ ) $\delta 187.0$ (C-8), 174.5 (C-5), 171.4 (C-6), 146.6 (C-10), 127.1 (C-9), 103.0 (C-7), 55.9 (C-15), 54.6 (C-11), 52.8 (C-2), 41.8 (C-4), 41.7 (C-3), 27.5 (C-12, C-17, C-18), (CCDC: 1050853, these data can be obtained free of charge via http://www.ccdc.cam.ac.uk/conts/retrieving.html (or from the CCDC, 12 Union Road, Cambridge CB2 1EZ, UK; Fax: +441223 336033; E-mail: deposit@ccdc.cam.ac.uk)).

Acknowledgments: P.A.C. thanks CONACYT for his graduate scholarship (370144/304476) and Dr. Luis D. Miranda UNAM for technical support with respect to X-ray experiments. R.G.-M is grateful for financial support from the DAIP (859/2016) and CONACYT (CB-2011-166747-Q) projects. A.I.-J. acknowledges QI-DQ-CBI-UAMI for his position as visiting professor (40966) and PRODEP for financial support (12413143).

Author Contributions: P.A.C. performed the thermal spirocyclization study; A.I.-J. wrote the paper; R.G.-M. is the responsible researcher and to whom correspondence must be addressed.

Conflicts of Interest: The authors declare no conflict of interest. The founding sponsors had no role in the design of the study; in the collection, analyses, or interpretation of data; in the writing of the manuscript, and in the decision to publish the results.

\section{References}

1. V. A. D'yakonov, O. A. Trapeznikova, A. D. Meijere, U. M. Dzhemilev, Chem. Rev. 2014, 114, 5775.

2. R. Ramon, Chem. Soc. Rev. 2012, 41, 1060.

3. H. B. Park, Y.-J. Kim, J. K. Lee, K. R. Lee, H. C. Kwon, Org. Lett. 2012, 14, 5002.

4. Y.-L. Yang, F.-R. Chang, Y.-C. Wu, Helv. Chim. Acta 2004, 87, 1392.

5. D. H. Hey, A. R. Todd, J. Chem. Soc. C 1967, 1518.

6. J. Boivin, M. Yousfi, S.Z. Zard, Tetrahedron Lett. 1997, 38, 5985.

7. F. Gonzalez-Lopez de Turiso, D.P. Curran, Org. Lett. 2005, 7, 151.

8. T.R. Ibarra-Rivera, R. Gámez-Montaño, L.D. Miranda, Chem. Commun. 2007, 3485.

9. C.R. Reddy, S.K. Prajapti, K. Warudikar, R. Ranjan, B.B. Rao, Org. Biomol. Chem. 2017, 15, 3130.

10. L.R. Ryzhkov, J. Org. Chem. 1996, 61, 2801.

(C) 2017 by the authors. Submitted for possible open access publication under the terms and conditions of the Creative Commons Attribution (CC BY) license (http://creativecommons.org/licenses/by/4.0/). 